\title{
Threshold Saturation on Channels with Memory via Spatial Coupling
}

\author{
Shrinivas Kudekar* and Kenta Kasai ${ }^{\dagger}$ \\ * New Mexico Consortium and Center for Non-linear Studies, Los Alamos National Laboratory, NM, USA \\ Email: skudekar@lanl.gov \\ $\dagger$ Dept. of Communications and Integrated Systems, Tokyo Institute of Technology, 152-8550 Tokyo, Japan. \\ Email: kenta@comm.ss.titech.ac.jp
}

\begin{abstract}
We consider spatially coupled code ensembles. A particular instance are convolutional LDPC ensembles. It was recently shown that, for transmission over the memoryless binary erasure channel, this coupling increases the belief propagation threshold of the ensemble to the maximum a-posteriori threshold of the underlying component ensemble. This paved the way for a new class of capacity achieving low-density parity check codes. It was also shown empirically that the same threshold saturation occurs when we consider transmission over general binary input memoryless channels.

In this work, we report on empirical evidence which suggests that the same phenomenon also occurs when transmission takes place over a class of channels with memory. This is confirmed both by simulations as well as by computing EXIT curves.
\end{abstract}

\section{INTRODUCTION}

It has long been known that convolutional LDPC (or spatially coupled) ensembles, introduced by Felström and Zigangirov [1], have excellent thresholds when transmitting over general binary-input memoryless symmetric-output (BMS) channels. The fundamental reason underlying this good performance was recently discussed in detail in [2] for the case when transmission takes place over the binary erasure channel (BEC).

In particular, it was shown in [2] that the BP threshold of the spatially coupled ensemble (see the last paragraph of this section for a definition) is essentially equal to the MAP threshold of the underlying component ensemble. It was also shown that for long chains the MAP performance of the chain cannot be substantially larger than the MAP threshold of the component ensemble. In this sense, the BP threshold of the chain is increased to its maximal possible value. This is the reason why they call this phenomena threshold saturation via spatial coupling. In a recent paper [3], Lentmaier and Fettweis independently formulated the same statement as conjecture. They attribute the observation of the equality of the two thresholds to G. Liva. The phenomena of threshold saturation seems not to be restricted to the BEC. It was also shown recently in [4] that the same phenomena manifests itself when we consider transmission over more general BMS channels.

The principle which underlies the good performance of spatially coupled ensembles is very broad. It has been shown to apply to many other problems in communications, and more generally computer science. To mention just a few, the threshold saturation effect (dynamical threshold of the system being equal to the static or condensation threshold) of coupled graphical models has recently been shown to occur for compressed sensing [5], and a variety of graphical models in statistical physics and computer science like the so-called $K$-SAT problem, random graph coloring, or the Curie-Weiss model [6]. Other communication scenarios where the spatially coupled codes have found immediate application is to achieve the whole rate-equivocation region of the BEC wiretap channel [7].

It is tempting to conjecture that the same phenomenon occurs for transmission over general channels with memory. We provide some empirical evidence that this is indeed the case. In particular, we compute EXIT curves for transmission over a class of channels with memory known as the Dicode Erasure Channel (DEC). We show that these curves behave in an identical fashion to the ones when transmission takes place over the memoryless BEC. We also compute fixed points (FPs) of the spatial configuration and we demonstrate again empirically that these FPs have properties identical to the ones in the BEC case.

For a review on the literature on convolutional LDPC ensembles we refer the reader to [2] and the references therein. As discussed in [2], there are many basic variants of coupled ensembles. For the sake of convenience of the reader, we quickly review the ensemble $\left(d_{1}, d_{\mathrm{r}}, L, w\right)$. This is the ensemble we use throughout the paper as it is the simplest to analyze.

\section{A. $\left(d_{1}, d_{\mathrm{r}}, L, w\right)$ Ensemble [2]}

We assume that the variable nodes are at sections $[-L, L]$, $L \in \mathbb{N}$. At each section there are $M$ variable nodes, $M \in$ $\mathbb{N}$. Conceptually we think of the check nodes to be located at all integer positions from $[-\infty, \infty]$. Only some of these positions actually interact with the variable nodes. At each position there are $\frac{d_{1}}{d_{\mathrm{r}}} M$ check nodes. It remains to describe how the connections are chosen. We assume that each of the $d_{1}$ connections of a variable node at position $i$ is uniformly and independently chosen from the range $[i, \ldots, i+w-1]$, where $w$ is a "smoothing" parameter. In the same way, we assume that each of the $d_{\mathrm{r}}$ connections of a check node at position $i$ is independently chosen from the range $[i-w+1, \ldots, i]$.

A discussion on the above ensemble and a proof of the following lemma can be found in [2]. 
Lemma 1 (Design Rate): The design rate of the ensemble $\left(d_{\mathrm{l}}, d_{\mathrm{r}}, L, w\right)$, with $w \leq 2 L$, is given by

$$
R\left(d_{\mathrm{l}}, d_{\mathrm{r}}, L, w\right)=\left(1-\frac{d_{\mathrm{l}}}{d_{\mathrm{r}}}\right)-\frac{d_{\mathrm{l}}}{d_{\mathrm{r}}} \frac{w+1-2 \sum_{i=0}^{w}\left(\frac{i}{w}\right)^{d_{\mathrm{r}}}}{2 L+1} .
$$

In the next section we provide the channel model and the joint iterative decoder. We also present the density evolution analysis of the joint iterative decoder when we consider $\left(d_{\mathrm{l}}, d_{\mathrm{r}}\right)$-regular LDPC ensembles. In the section on main results, we demonstrate the threshold saturation phenomena by using spatially coupled codes.

\section{Channels with Memory: The Dicode Erasure CHANNEL}

The particular class of channel with memory that we consider is the Dicode Erasure Channel (DEC). The DEC is a binary-input channel defined as follows. The output of a binary-input linear filter $(1-D)$ ( $D$ is the delay element) is erased with probability $\epsilon$ and transmitted perfectly with probability $1-\epsilon$. For this channel we will be interested in the symmetric information rate (SIR), i.e., the capacity assuming i.i.d Bern(1/2) signalling. In this case, the Shannon threshold for a given rate $r$ is given by $\frac{1-r}{4}+\frac{1}{4} \sqrt{(1-r)^{2}+8(1-r)}$. The details on the definition of the channel and the analytical formula for the SIR can be found in the thesis of Pfister [8] and in [9].

\section{A. Joint Iterative Decoder, Density Evolution and the Ex- tended BP Fixed Points}

We use the joint iterative decoder (JIT) of Pfister and Siegel [9]. More precisely, we consider a turbo equalization system, which performs one channel iteration (BCJR step) for each iteration over the LDPC code. As a result, in every iteration, first the channel detector uses the extrinsic information provided by the LDPC code to compute its extrinsic erasure fraction. This is then fed to the LDPC decoder which then again computes the usual variable node and check node erasure messages.

The simplicity of the DEC gives an analytical formula for the erasure fraction of the message which is passed from the channel detector to the LDPC code (see [9] for a derivation). This is given by

$$
f(x)=\frac{4 \epsilon^{2}}{(2-x(1-\epsilon))^{2}},
$$

where $x$ represents the fraction of erasures entering the channel detector from the LDPC code. $f($.$) represents the extrinsic$ erasure information provided by the channel detector.

To summarize: the density evolution 1 (DE) equation for the case of $\left(d_{l}, d_{\mathrm{r}}\right)$-regular LDPC ensemble is given by

$$
x=f\left(\left(1-(1-x)^{d_{\mathrm{r}}-1}\right)^{d_{\mathrm{l}}}\right)\left(1-(1-x)^{d_{\mathrm{r}}-1}\right)^{d_{\mathrm{l}}-1} .
$$

Note that the term inside the brackets in $f($.$) represents the$ probability that a variable node is in erasure as given by the LDPC code. Also it is not hard to see that $f(x) \leq 1$ for any $x$.

\footnotetext{
${ }^{1}$ See [9] for a rigorous justification of the density evolution analysis.
}

Example 2: Consider JIT decoding of the DEC with $(5,15)$-regular LDPC ensemble. The design rate of this code is $2 / 3$. Using the SIR formula $\left(=1-2 \epsilon^{2} /(1+\epsilon)\right.$ ) from [9] we get that the Shannon threshold at rate $=2 / 3$ is given by $\epsilon_{\mathrm{DEC}}^{\mathrm{Sh}}=0.5$. Figure 1 shows the performance of the JIT decoder. We see that the threshold is given by $\epsilon_{\mathrm{DEC}}^{\mathrm{IT}}(5,15) \approx 0.363471$, which is far away from the capacity. Throughout the paper we will use $\epsilon_{\mathrm{DEC}}^{\mathrm{IT}}\left(d_{\mathrm{l}}, d_{\mathrm{r}}\right)$ to denote the threshold of the JIT decoder when we use $\left(d_{1}, d_{\mathrm{r}}\right)$-regular LDPC ensemble and transmit over the DEC.

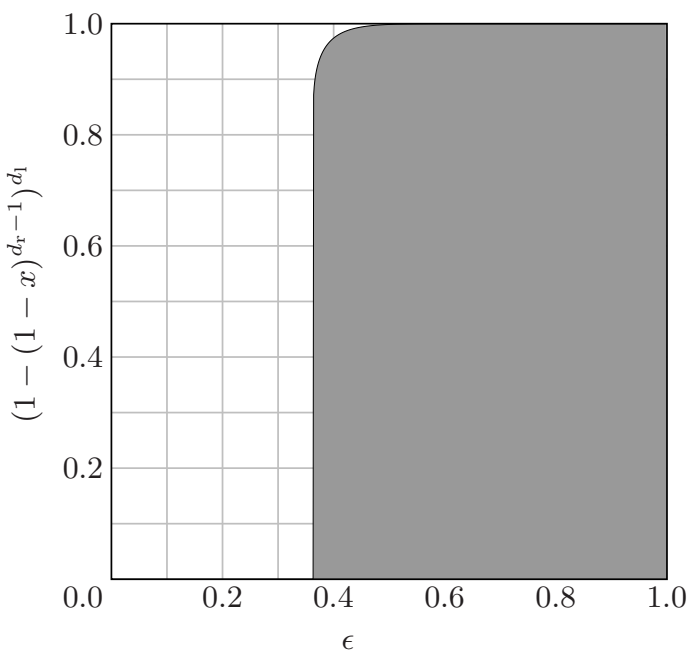

Fig. 1. The BP curve for the $\left(d_{1}=5, d_{\mathrm{r}}=15\right)$-regular ensemble and transmission over the DEC. The threshold of the JIT decoder is given by $\epsilon_{\mathrm{DEC}}^{\mathrm{ITT}}(5,15) \approx 0.363471$.

The EXIT curve: The EXIT curve 2 plots all the fixed-points of the DE equation. The curve is given by the parametric curve $\left\{\left(1-(1-x)^{d_{\mathrm{r}}-1}\right)^{d_{1}}, \epsilon(x)\right\}$. We obtain $\epsilon(x)$ by solving for $\epsilon$ in the DE equation.

As an example, we plot the EXIT curve for various $\left(d_{l}, d_{\mathrm{r}}\right)$ regular LDPC ensembles as shown in Figure 2. The JIT threshold is got by dropping a vertical line from the leftmost point on any given curve. We note that for every $\epsilon>\epsilon_{\mathrm{DEC}}^{\mathrm{IT}}\left(d_{\mathrm{l}}, d_{\mathrm{r}}\right)$, there are exactly 3 fixed-points. One of them being the trivial 0 fixed-point. This " $C$ " shape of the EXIT curve is also what we observe when we transmit through a memoryless BEC using $\left(d_{l}, d_{\mathrm{r}}\right)$-regular LDPC ensemble. Also we remark that as the degrees increase, keeping the design rate fixed, the JIT threshold keeps on decreasing. This is also the case for transmission over memoryless BEC. In fact, for memoryless BEC case, the BP threshold goes to zero as we increase the degrees. We can also show the same result for the DEC. More precisely, we have

Lemma 3 (JIT Threshold Goes to Zero): For any $\left(d_{1}, d_{\mathrm{r}}\right)$ regular ensemble we have

$$
\epsilon_{\mathrm{DEC}}^{\mathrm{ITT}}\left(d_{\mathrm{l}}, d_{\mathrm{r}}\right) \leq \sqrt{\frac{1}{\sqrt{d_{\mathrm{r}}-1}\left(1-\left(d_{\mathrm{l}}-1\right) e^{-\sqrt{d_{\mathrm{r}}-1}}\right)}} .
$$

${ }^{2}$ To be very precise, we should call the curves we plot as EXIT-like curves. The reason being that we do not provide any operation interpretation of these curves, like the Area theorem [10] in this work. The curves serve only to illustrate the capacity achieving nature of coupled-codes. 


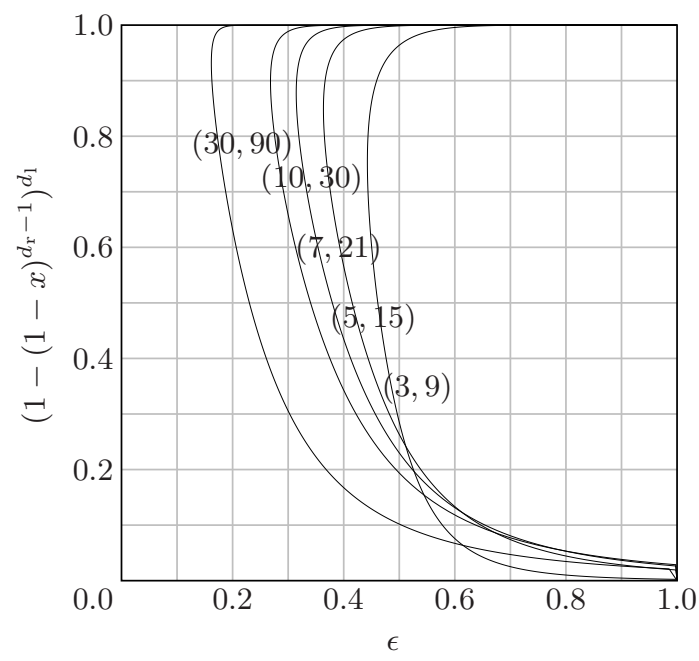

Fig. 2. The EXIT curve for regular LDPC ensembles with $\left(d_{1}, d_{\mathrm{r}}\right)$ given by $(3,9),(5,15),(7,21),(10,30),(30,90)$, and transmission over the DEC. We observe that the JIT threshold moves to the left and eventually will go to zero as degrees go to infinity.

Proof: We claim that the necessary condition for the JIT decoder to succeed is given by

$$
\epsilon^{2}\left(1-(1-x)^{d_{\mathrm{r}}-1}\right)^{d_{1}-1}<x,
$$

for all $x \in(0,1]$. Indeed, suppose on the contrary that there exists a $c \in(0,1]$ such that the above inequality is violated. Thus we have $\epsilon^{2}\left(1-(1-c)^{d_{r}-1}\right)^{d_{1}-1} \geq c$. Since $f(x) \geq \epsilon^{2}$ for all $x \in[0,1]$ we get

$$
f(c)\left(1-(1-c)^{d_{\mathrm{r}}-1}\right)^{d_{1}-1} \geq c .
$$

This implies that there exists a FP of DE for the DEC for some value in $[c, 1]$. It is not hard to see that this implies the JIT decoder will get stuck at this FP, resulting in unsuccessful decoding.

Thus we must have that for all $x \in(0,1]$

$$
\epsilon^{2}\left(1-(1-x)^{d_{\mathrm{r}}-1}\right)^{d_{1}-1}<x .
$$

For the choice of $x=\frac{1}{\sqrt{d_{\mathrm{r}}-1}}$ we get the statement of the lemma. To see this computation first write $(1-x)^{d_{\mathrm{r}}-1}$ as $e^{\left(d_{\mathrm{r}}-1\right) \log (1-x)}$. Then use $\log (1-x) \leq-x$ and $x=\frac{1}{\sqrt{d_{\mathrm{r}}-1}}$ to get $(1-x)^{d_{\mathrm{r}}-1} \leq e^{-\sqrt{d_{\mathrm{r}}-1}}$. After this use

$$
\begin{aligned}
\left(1-e^{-\sqrt{d_{\mathrm{r}}-1}}\right)^{d_{1}-1} & =1-\left(1-\left(1-e^{-\sqrt{d_{\mathrm{r}}-1}}\right)^{d_{1}-1}\right) \\
& \geq 1-\left(d_{\mathrm{l}}-1\right) e^{-\sqrt{d_{\mathrm{r}}-1}},
\end{aligned}
$$

to complete the argument.

As a consequence of Lemma 3 we get that, with the ratio $d_{\mathrm{l}} / d_{\mathrm{r}}$ kept fixed, $\lim _{d_{\mathrm{l}} \rightarrow \infty} \epsilon_{\mathrm{DEC}}^{\mathrm{IT}}\left(d_{\mathrm{l}}, d_{\mathrm{r}}\right)=0$.

\section{Main Results}

In this section we show, empirically, that spatially coupledcodes achieve the Shannon capacity of the DEC. We recall that we are consider SIR which is give by the formula SIR= $1-2 \epsilon^{2} /(1+\epsilon)$. For the sake of exposition, we demonstrate our results only for rate equals $2 / 3$. The Shannon threshold for this rate is given by $\epsilon_{\mathrm{DEC}}^{\mathrm{Sh}}=0.5$. For other rates similar results can be observed. From the preceding section we see that standard $\left(d_{\mathrm{l}}, d_{\mathrm{r}}\right)$-regular LDPC ensembles do not saturate the JIT threshold (to the Shannon threshold).

We begin by writing down the DE equation for the coupledcodes.

\section{A. Density Evolution}

Consider the $\left(d_{\mathrm{l}}, d_{\mathrm{r}}, L, w\right)$ ensemble. Recall that there are $2 L+1$ sections of variable nodes. Each section has $M$ variable nodes. We transmit variable nodes sectionwise over the DEC. More precisely, the variable nodes in section $-L$ are transmitted first, followed by variable nodes in section $-L+1$ and so on so forth till we finally transmit all the variable node in section $L$. As a consequence we have a channel detected factor graph sitting on top of each section of the coupled-code.

To perform the DE analysis, we already take the limit $M \rightarrow$ $\infty$. As a result of this limit, one can ignore the boundary effects of the channel detector and treat the channel detectors as disconnected.

Let $x_{i}, i \in \mathbb{Z}$, denote the average erasure probability which is emitted by variable nodes at position $i$. For $i \notin[-L, L]$ we set $x_{i}=0$. For $i \in[-L, L]$ the $\mathrm{DE}$ is given by

$$
x_{i}=\epsilon_{i}\left(1-\frac{1}{w} \sum_{j=0}^{w-1}\left(1-\frac{1}{w} \sum_{k=0}^{w-1} x_{i+j-k}\right)^{d_{\mathrm{r}}-1}\right)^{d_{1}-1},
$$

where $\epsilon_{i}$ is given by

$$
\epsilon_{i}=f\left(\left(1-\frac{1}{w} \sum_{j=0}^{w-1}\left(1-\frac{1}{w} \sum_{k=0}^{w-1} x_{i+j-k}\right)^{d_{\mathrm{r}}-1}\right)^{d_{1}}\right),
$$

where recall that $f(\cdot)$ is the channel extrinsic transfer function. We will use the notation $\epsilon_{\mathrm{DEC}}^{\mathrm{JT}}\left(d_{\mathrm{l}}, d_{\mathrm{r}}, L, w\right)$ to denote the threshold of the JIT decoder when we use the $\left(d_{1}, d_{\mathrm{r}}, L, w\right)$ ensemble for transmission. As a shorthand we use $g\left(x_{i-w+1}, \ldots, x_{i+w-1}\right)$ to denote $\left(1-\frac{1}{w} \sum_{j=0}^{w-1}(1-\right.$ $\left.\left.\frac{1}{w} \sum_{k=0}^{w-1} x_{i+j-k}\right)^{d_{\mathrm{r}}-1}\right)^{d_{1}-1}$.

Definition 4 (FPs of Density Evolution): Consider DE for the $\left(d_{\mathrm{l}}, d_{\mathrm{r}}, L, w\right)$ ensemble. Let $\underline{x}=\left(x_{-L}, \ldots, x_{L}\right)$. We call $\underline{x}$ the constellation. We say that $\underline{x}$ forms a FP of DE with channel $\epsilon$ if $\underline{x}$ fulfills $(1)$ for $i \in[-L, L]$. As a shorthand we then say that $(\epsilon, \underline{x})$ is a FP. We say that $(\epsilon, \underline{x})$ is a non-trivial FP if $\underline{x}$ is not identically equal to $0 \forall i$. Again, for $i \notin[-L, L]$, $x_{i}=0$.

Definition 5 (Forward DE and Admissible Schedules):

Consider forward DE for the $\left(d_{1}, d_{\mathrm{r}}, L, w\right)$ ensemble. More precisely, pick a channel $\epsilon$. Initialize $\underline{x}^{(0)}=(1, \ldots, 1)$. Let $\underline{x}^{(\ell)}$ be the result of $\ell$ rounds of DE. More precisely, $\underline{x}^{(\ell+1)}$ is generated from $\underline{x}^{(\ell)}$ by applying the DE equation (11) to each section $i \in[-\bar{L}, L]$,

$$
x_{i}^{(\ell+1)}=\epsilon_{i} g\left(x_{i-w+1}^{(\ell)}, \ldots, x_{i+w-1}^{(\ell)}\right) .
$$

\footnotetext{
${ }^{3}$ Another way to think about this is to imagine that we transmit a known sequence of bits of length equal to the memory of the channel after we transmit all the variable nodes in each section. Since the channel memory is finite, this induces a rate loss going to zero as $M \rightarrow \infty$. Now the known sequence is the initial state for each of the channel detectors and hence we can consider them disconnected.
} 
We call this the parallel schedule. The important difference with the memoryless BEC case is that the channel $\epsilon_{i}$ is not fixed for the DEC and decreases with increasing iterations according to (2).

More generally, consider a schedule in which in each step $\ell$ an arbitrary subset of the sections is updated, constrained only by the fact that every section is updated in infinitely many steps. We call such a schedule admissible. Again, we call $\underline{x}^{(\ell)}$ the resulting sequence of constellations.

One can show that if we perform forward DE under any admissible schedule, then the constellation $\underline{x}^{(\ell)}$ converges to a FP of DE and this FP is independent of schedule. This statement can be proved similar to the one in [2].

\section{B. Forward DE - Simulation Results}

We consider forward DE for the $\left(d_{1}, d_{\mathrm{r}}, L, w\right)$ ensemble. More precisely, we fix an $\epsilon$ and initialize all $x_{i}$ for $i \in[-L, L]$ to 1 . Then we run the DE given by (1) till we reach a fixedpoint. We fix $L=250$. For $d_{1}=3$ and $d_{\mathrm{r}}=9$, we have that $\epsilon_{\mathrm{DEC}}^{\mathrm{ITT}}(3,9,300,3) \approx 0.49815$. If we increase the degrees we get $\epsilon_{\mathrm{DEC}}^{\mathrm{ITT}}(5,15,300,5) \approx 0.49995, \epsilon_{\mathrm{DEC}}^{\mathrm{ITT}}(7,21,300,7) \approx$ 0.499989 and $\epsilon_{\mathrm{DEC}}^{\mathrm{IT}}(9,27,300,9) \approx 0.499996$. We observe that for increasing the degrees the threshold approaches the Shannon threshold of 0.5 .

\section{The EXIT Curve for Coupled Ensembles}

We now come to the key point of the paper, the computation of the EXIT curve. Before we do this, we define the entropy of a constellation $\underline{x}=\left(x_{-L}, \ldots, x_{L}\right)$ as

$$
\chi=\frac{1}{2 L+1} \sum_{i=-L}^{L} x_{i} .
$$

To plot the EXIT curve we first fix $\chi \in[0,1]$ and then run DE such that the resulting FP constellation has entropy equal to $\chi$. This is the reverse DE procedure as described in [11]. We remark that $f(x)$ is an increasing function of $\epsilon$, hence in the reverse DE procedure one can easily find an appropriate $\epsilon$ by the bisection method.

Figure 3 shows the plot of the EXIT curve for the $(5,15, L, 5)$ ensemble with $L=$ $2,4,8,16,32,64,128,256,512$. We see that the curves look very similar to the curves when transmitting over a BMS channel. For very small values of $L$, the curves are far to the right due to significant rate loss that is incurred at the boundary. As $L$ increases the rate loss diminishes and the JIT threshold is very close to the Shannon threshold. This picture strongly suggests that the same threshold saturation effect $\left(\epsilon_{\mathrm{DEC}}^{\mathrm{IT}}\left(d_{1}, d_{\mathrm{r}}, L, w\right) \approx \epsilon_{\mathrm{DEC}}^{\mathrm{MAP}}\left(d_{1}, d_{\mathrm{r}}, L, w\right)\right)$ also occurs for the DEC as it was shown analytically in [2].

\section{Shape of Fixed Point of Density Evolution}

We plot the constellation representing the unstable FP of DE. This FP cannot be reached via forward DE and is obtained via reverse DE procedure. We recall that this FP played a key role in proving the threshold saturation phenomena when

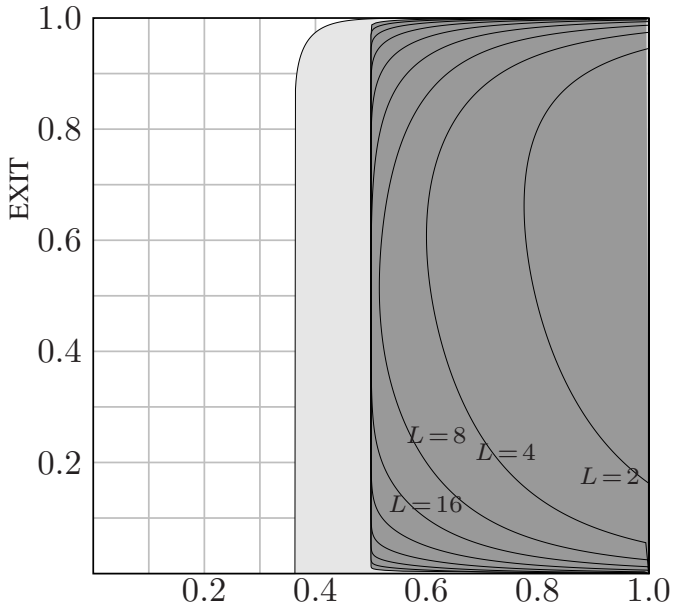

Fig. 3. The EXIT curve for the $\left(d_{1}=5, d_{\mathrm{r}}=15, L, 5\right)$ ensemble and transmission over the DEC for $L=2,4,8,16,32,64,128,256,512$. The curves keep moving to the left as $L$ increases similar to the curves when transmitting over BMS. The "vertical" drop in the EXIT curves occurs at $\approx 0.5$ for $L \geq 32$. Also shown in light gray is the BP exit curve for the uncoupled $(5,15)$-regular ensemble.

transmitting over the BEC. Let us describe the (empirically observed) crucial properties of this constellation.

(i) The constellation is symmetric around $i=0$ and is unimodal. The constellation has $\epsilon \approx 0.49995$.

(ii) Let $x_{\mathrm{s}}(\epsilon)$ denote a stable $\mathrm{FP}$ of $\mathrm{DE}$. The value in the flat part in the middle is $\approx 0.4434$ which is very close to the stable FP of DE for the underlying uncoupled $(5,15)$ regular ensemble.

(iii) The transition from close to zero to close to $x_{\mathrm{s}}(\epsilon)$ is very quick.

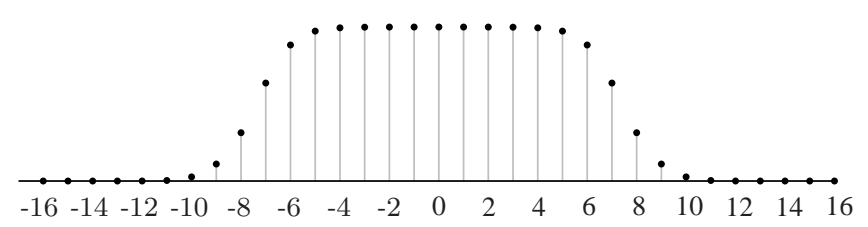

Fig. 4. The constellation representing FP of DE for $(5,15,33,5)$ ensemble and entropy fixed to $\chi=0.2$. This is an unstable FP constellation. The constellation is very similar to any unstable FP constellation when transmitting over memoryless BEC. The constellation is unimodal. There is a long tail of zeros followed by a sharp transition and then a long flat part with values close to $x_{\mathrm{s}}(\epsilon)$. The constellation has $\epsilon \approx 0.49995$.

\section{A Possible Proof ApproAch}

Till now we gave empirical evidence of the threshold saturation phenomena when transmitting over the DEC using coupled-codes. Before we proceed to give the proof idea for the threshold saturation, we first show that coupling indeed helps. More precisely we have the following lemma,

Lemma 6 (Spatial Coupling Helps): For $d_{1}, d_{\mathrm{r}} \rightarrow \infty$ with the ratio $d_{\mathrm{l}} / d_{\mathrm{r}}$ kept fixed, we have

$$
\epsilon_{\mathrm{DEC}}^{\mathrm{ITT}}\left(d_{\mathrm{l}}, d_{\mathrm{r}}, L, w\right) \geq \frac{d_{\mathrm{l}}}{d_{\mathrm{r}}}
$$


Proof: Since $\epsilon_{i}$ is an increasing function of $x_{i-w+1}, \ldots, x_{i+w-1}$, we have $\epsilon_{i} \leq f(1) \leq \frac{4 \epsilon^{2}}{(1+\epsilon)^{2}} \leq \epsilon$. Combining this with the DE equation for the coupled-codes, we get

$$
x_{i} \leq \epsilon g\left(x_{i-w+1}, \ldots, x_{i+w-1}\right),
$$

for all $i \in[-L, L]$. But we know from Theorem 10 in [2] that $\lim _{d_{1} \rightarrow \infty} \epsilon_{\mathrm{BEC}}^{\mathrm{BP}}\left(d_{1}, d_{\mathrm{r}}, L, w\right) \rightarrow \frac{d_{1}}{d_{\mathrm{r}}}$. Thus for $\epsilon<\frac{d_{1}}{d_{\mathrm{r}}}$ the right-hand-side of the above inequality goes to zero. Hence the lemma.

As an example, consider the $\left(d_{\mathrm{l}}, d_{\mathrm{r}}\right)$-regular ensemble with $d_{1} / d_{\mathrm{r}}=1 / 3$ (rate equal to $2 / 3$ ). For $L \rightarrow \infty$, the rate of the $\left(d_{\mathrm{l}}, d_{\mathrm{r}}, L, w\right)$ goes to $2 / 3$. From Lemma 3 we have that $\epsilon_{\mathrm{DEC}}^{\mathrm{ITT}}\left(d_{\mathrm{l}}, d_{\mathrm{r}}\right) \rightarrow 0$ and from Lemma 6 we have that $\epsilon_{\mathrm{DEC}}^{\mathrm{ITT}}\left(d_{\mathrm{l}}, d_{\mathrm{r}}, L, w\right) \geq \frac{d_{\mathrm{l}}}{d_{\mathrm{r}}}=\frac{1}{3}$. Thus spatial coupling indeed boosts the JIT threshold. However the empirical evidence suggests that the boost is all the way up to the Shannon threshold (which is 0.5 in this case). Since there is ample similarity between the DEC and the BEC, the guideline for a proof is similar to when we are transmitting over the BEC.

(i) Existence of FP: A key ingredient in proving the result for the BEC was to show the existence of a special FP of DE $\left(\underline{x}, \epsilon^{*}\right)$. In principle, the BEC proof should extend. The only difference is that instead of a constant channel $\epsilon$, we have a channel value which depends on the FP constellation itself. However, since the functions involved are rational, this should not be a big hurdle.

(ii) Shape of the constellation and the transition length: The next task is to show that the FP guaranteed by the above theorem has the properties as given in Section [II-D Proving this would first involve showing that the underlying regular ensemble has a "C" shaped EXIT curve. Intuitively, this means that the FP constellation (of the coupled-code) can only hover around the stable FPs of DE (of the underlying regular ensemble), implying that it has either a large tail of zeros or a large flat part with values close to $x_{\mathrm{s}}\left(\epsilon^{*}\right)$.

(iii) Construction of the EXIT curve and the Area Theorem: Another key part of the BEC proof was to construct a family of FPs (not necessarily stable FPs) using the special FP guaranteed by the Existence theorem. The EXIT curve plus the fast transition would allow us to show that this special FP must have an associated channel parameter, $\epsilon^{*}$, very close to the Shannon threshold (for large degrees. $)^{4}$

Operational interpretation: The proof would be completed by providing an operation meaning to the EXIT curve. Loosely speaking, the EXIT constructed above would have a vertical drop at $\epsilon \approx \epsilon^{\mathrm{Sh}}\left(d_{\mathrm{l}}, d_{\mathrm{r}}\right)$ (cf. Figure 3). This would help to show that for any $\epsilon<\epsilon^{\mathrm{Sh}}\left(d_{1}, d_{\mathrm{r}}\right)$, the JIT decoder will go to the trivial FP.

\section{CONCLUSiOnS}

In this paper we show that empirically coupled-codes saturate the JIT threshold on the DEC. For the channel extrinsic

\footnotetext{
${ }^{4}$ For finite degrees, $\epsilon^{*}$ should be very close to the MAP threshold of the $\left(d_{1}, d_{\mathrm{r}}\right)$-regular ensemble. One should be able to prove this by formulating an appropriate Area theorem (see Section 3.20 in [10]).
}

transfer function we consider the case when there is no precoding. We list below some comments and open questions.

(i) An obvious future direction is to complete the proof of threshold saturation. The guidelines provided above serve as a starting point. Following this route, in principle, it should be possible to prove the capacity achieving nature of these codes on the DEC.

(ii) Another interesting question is that whether the threshold saturation phenomena can be shown to be true for all channel extrinsic transfer functions $f($.$) which are non-$ decreasing both in $\epsilon$ and $x$ (threshold saturation holds when $f($.$) represents precoding).$

(iii) A proof of the threshold saturation phenomena should also pave the way for the justification of the Maxwell construction to determine $\epsilon_{\mathrm{DEC}}^{\mathrm{MAP}}\left(d_{1}, d_{\mathrm{r}}\right)$ for the DEC.

(iv) Recently, it was observed that coupled MacKay-Neal (MN) codes with bounded degree exhibit the BP threshold very close to the Shannon threshold over the BEC [12]. It is interesting to see if the coupled MN codes have the JIT threshold close to the SIR over the DEC.

\section{ACKNOWLEDGMENTS}

SK acknowledges support of NMC via the NSF collaborative grant CCF-0829945 on "Harnessing Statistical Physics for Computing and Communications." SK would also like to thank Rüdiger Urbanke, Misha Chertkov and Henry Pfister for their encouragement.

\section{REFERENCES}

[1] A. J. Felström and K. S. Zigangirov, "Time-varying periodic convolutional codes with low-density parity-check matrix," IEEE Trans. Inform. Theory, vol. 45, no. 5, pp. 2181-2190, Sept. 1999.

[2] S. Kudekar, T. Richardson, and R. Urbanke, "Threshold saturation via spatial coupling: Why convolutional LDPC ensembles perform so well over the BEC," 2010, e-print: http://arxiv.org/abs/1001.1826.

[3] M. Lentmaier and G. P. Fettweis, "On the thresholds of generalized LDPC convolutional codes based on protographs," in Proc. of the IEEE Int. Symposium on Inform. Theory, Austing, TX, USA, June 2010, pp. 709-713.

[4] S. Kudekar, C. Méasson, T. Richardson, and R. Urbanke, "Threshold saturation on BMS channels via spatial coupling," Apr. 2010, e-print: http://arxiv.org/abs/1004.3742.

[5] S. Kudekar and H. D. Pfister, "The effect of spatial coupling on compressive sensing," in Proc. of the Allerton Conf. on Commun., Control, and Computing, Monticello, IL, USA, 2010.

[6] S. H. Hassani, N. Macris, and R. Urbanke, "Coupled graphical models and their thresholds," in Proc. of the IEEE Inform. Theory Workshop, Dublin, Ireland, Sept. 2010.

[7] V. Rathi, R. Urbanke, M. Andersson, and M. Skoglund, "Rateequivocation optimally spatially coupled LDPC codes for the BEC wiretap channel," 2010, e-print: http://arxiv.org/abs/1010.1669.

[8] H. D. Pfister, "On the capacity of finite state channels and the analysis of convolutional accumulate- $m$ codes," Ph.D. dissertation, UCSD, San Diego, CA, USA, 2003.

[9] H. D. Pfister and P. H. Siegel, "Joint iterative decoding of LDPC codes for channels with memory and erasure noise," IEEE J. Sel. Area. Commun., vol. 26, no. 2, pp. 320-337, Feb. 2008.

[10] T. Richardson and R. Urbanke, Modern Coding Theory. Cambridge University Press, 2008.

[11] C. Méasson, A. Montanari, T. Richardson, and R. Urbanke, "The generalized area theorem and some of its consequences," IEEE Trans. Inform. Theory, vol. 55, no. 11, pp. 4793-4821, Nov. 2009.

[12] K. Kasai and K. Sakaniwa, "Spatially-coupled bounded-density capacity-achieving codes," in Proc. Symp. on Inf. Theory and its Applications, Dec. 2010, pp. 1-6, (in Japanese). 